EPJ Web of Conferences 108, 02045 (2016)

DOI: $10.1051 /$ epjconf/201610802045

(C) Owned by the authors, published by EDP Sciences, 2016

\title{
Ab Initio Investigations of Thermoelectric Effects in Graphene - Boron Nitride Nanoribbons
}

\author{
Camelia Visan ${ }^{1}$ and G. A. Nemnes ${ }^{1,2, a}$ \\ ${ }^{1}$ Horia Hulubei National Institute for Physics and Nuclear Engineering (IFIN-HH), 077126 Magurele-Ilfov, \\ Romania. \\ ${ }^{2}$ University of Bucharest, Faculty of Physics, Materials and Devices for Electronics and Optoelectronics \\ Research Center, P.O. Box MG-11, 077125 Magurele-llfov, Romania.
}

\begin{abstract}
Thermoelectric effects of graphene - hexagonal boron nitride (hBN) nanoribbons have been investigated by density functional theory (DFT) calculations. Pristine zig-zag nanoribbons are not suited to achieve high thermopower as the transmission function is flat around the chemical potential. By introducing $\mathrm{hBN}$ inclusions, the nanoribbon systems exhibit enhanced thermopower, due to the asymmetries introduced in the spin dependent transmission functions. Finite temperature differences between the two contacts are considered. The possibility of a good integration of hBN into graphene, makes the hybrid systems suitable for thermoelectric applications, which may be subject to further optimizations.
\end{abstract}

\section{Introduction}

Thermoelectric elements are important in a wide number of applications, ranging from energy conversion to cooling devices. In particular, thermoelectric generators (TG) are interesting, as they can convert the waste heat into electricity. Despite the present high cost-efficiency rate, the TGs have the advantage of being robust, with no moving parts and quite independent of the external environment. They are currently used e.g. in the automotive industry, as electricity sources along gas pipe-lines in remote areas and in medical use as cardio-stimulators, while radioisotope thermoelectric generators (RTGs) are indispensable in deep-space missions [1].

To achieve highly efficient TGs the active region should have a large electrical conductivity, a large Seebeck coefficient and low thermal conductivity, parameters which make up the thermoelectric figure of merit. Graphene is a material with high electron mobility, but it has also well known drawbacks with respect to thermoelectric applications [2]. Namely, the rather similar contributions of electrons and holes near the Fermi energy limits the Seebeck coefficient and the exceptionally high thermal conductivity [3] is not desired in this context.

Several approaches have been proposed for enhancing the thermoelectric properties of graphene. In order to decrease the thermal conductance, defect engineering may be employed, which is further influenced by the applied stress and by the choice of the substrate. Nanoribbons of different shapes [4], single molecule and quantum-dot nanojunctions [5], nanomeshes [6] have been considered in order

\footnotetext{
ae-mail: nemnes@theory.nipne.ro
} 


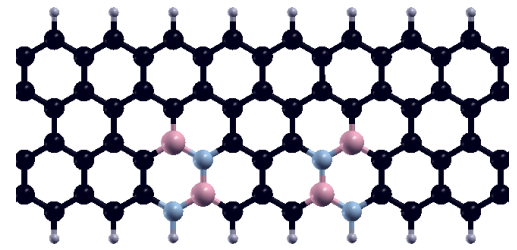

(a)

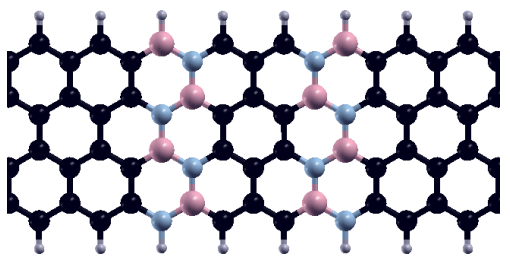

(c)

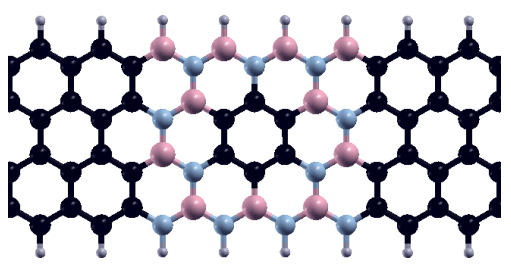

(e)

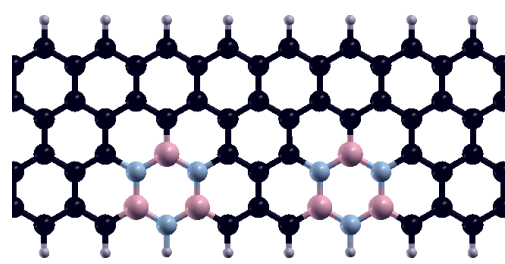

(b)

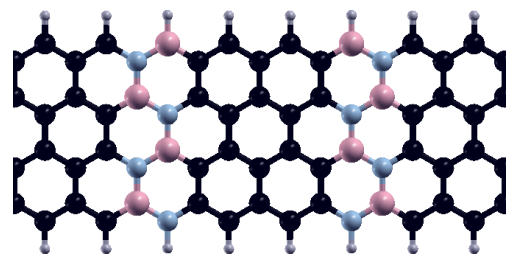

(d)

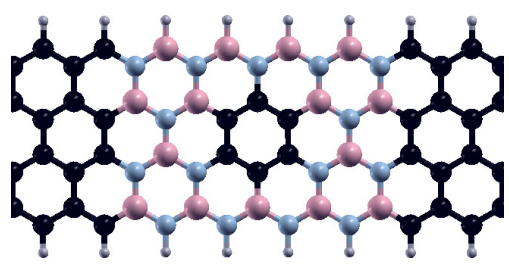

(f)

Figure 1. Hybrid graphene - hexagonal boron nitride zig-zag nanoribbons: thin and thick lateral barriers (a,b), tunneling double barrier systems $(\mathrm{c}, \mathrm{d})$ and graphene dots $(\mathrm{e}, \mathrm{f})$. The edges are hydrogen passivated.

to reduce the thermal conductivity. Furthermore, graphene allotropes [7-9] and hybrid graphene hexagonal boron nitride (hBN) structures may provide alternative routes.

It was recently demonstrated that atomic sized mixtures of graphene and $\mathrm{hBN}$ can be fabricated [10-12]. The two materials have a similar structure, but quite different electronic properties. While graphene is a zero gap semi-metal, $\mathrm{hBN}$ is a wide band gap semiconductor. Several applications using the hybrid graphene-hBN (G-hBN) materials were proposed, such as transistors [13], double barrier resonant tunneling devices [14, 15], spin current filters and switches [16-18], systems with tunable optical properties [19] and thermoelectric devices [20-22].

Here we investigate different configurations of hBN patterns in graphene nanorribons using spin polarized density functional theory (DFT) calculations. The analysis is performed taking into account finite temperature differences between the contacts, aiming for an optimum configuration of the GhBN domains.

\section{Computational model and method}

The model systems are zig-zag graphene nanoribbons with embedded hBN domains as indicated in Fig. 1. They are constructed from pristine graphene nanoribbons by progressively embedding $\mathrm{hBN}$ domains. The six structures considered here can be grouped into three categories, namely structures with lateral barriers $(a, b)$, tunneling double barrier systems $(c, d)$ and graphene dots $(e, f)$. The edges 

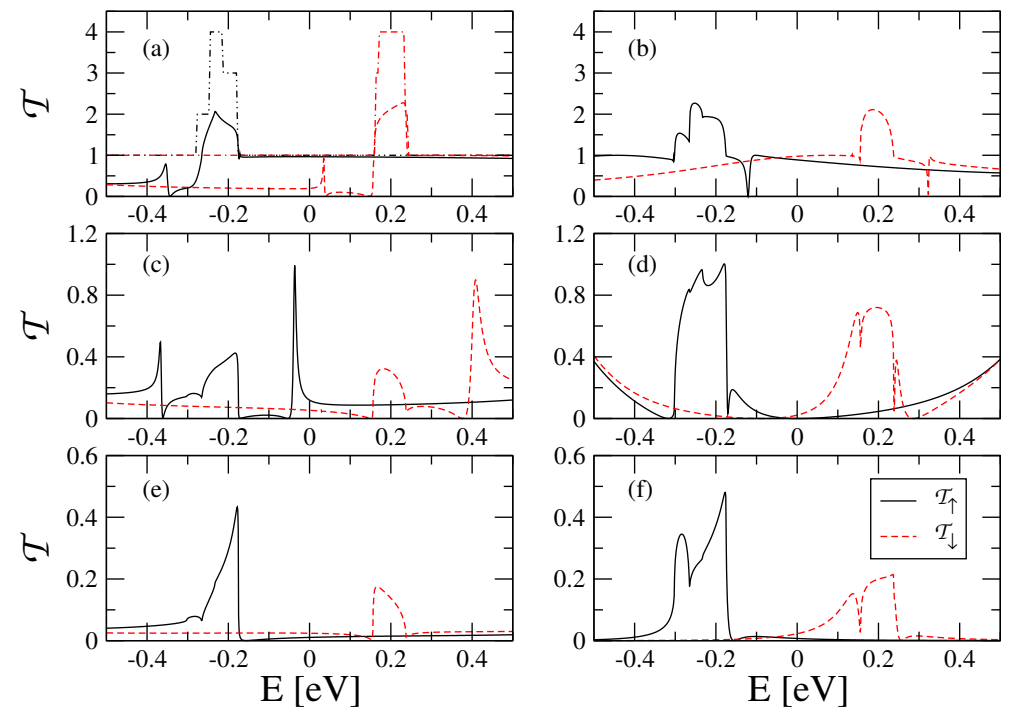

Figure 2. Spin dependent transmission functions for the structures depicted in Fig. 1(a-e). In plot (a) the ideal transmissions of the pristine graphene nanoribbon, $\mathcal{T}_{\uparrow}$ (dot-dot-dashed) and $\mathcal{T}_{\downarrow}$ (dash-dash-dotted), are depicted.

are hydrogen passivated. The semi-infinite electrodes consist of graphene nanoribbons of the same width, extending the outer left/right regions.

The $a b$ initio calculations are performed using the SIESTA package [23], which makes use of numeric atomic orbitals as basis set. The finite support of the basis elements ensure a linear scaling of the computational time with the system size. We make use of spin local density approximation (LSDA) in the parametrization of Ceperley and Alder. The real space grid is set by a mesh-cutoff of $100 \mathrm{Ry}$. The integrations in the reciprocal space were performed using a Monkhorst-Pack grid of $1 \times 1 \times 10$ k-points.

The spin dependent transmission functions $\mathcal{T}_{\uparrow / \downarrow}$ are calculated in the non-equilibrium Green's functions (NEGF) formalism implemented in TRANSIESTA [24]. The charge current is calculated by the Landauer formula:

$$
I=\frac{e}{h} \int d E \mathcal{T}(E)\left[f_{\mathrm{FD}}\left(E ; T_{\mathrm{L}}, \mu_{\mathrm{L}}\right)-f_{\mathrm{FD}}\left(E ; T_{\mathrm{R}}, \mu_{\mathrm{R}}\right)\right]
$$

where $\mathcal{T}=\mathcal{T}_{\uparrow}+\mathcal{T}_{\downarrow}$ is the total transmission function, $f_{\mathrm{FD}}$ is the Fermi-Dirac distribution function, $T_{\mathrm{L} / \mathrm{R}}$ and $\mu_{\mathrm{L} / \mathrm{R}}$ are the temperatures and electrochemical potentials in the left and right contacts, respectively. The applied bias is defined by $e U=\mu_{2}-\mu_{1}$.

The Seebeck coefficient (thermopower) $S$ is calculated for each pair of temperatures $\left(T_{\mathrm{L}}, T_{\mathrm{R}}\right)$ and, in the case of the open circuit condition $(I=0)$, it may be written as : $S_{\mathrm{oc}}=U_{\mathrm{oc}} / \Delta T$, where $\Delta T=T_{\mathrm{L}}-$ $T_{\mathrm{R}}$. However an important quantity for applications is the thermopower near the optimum working point, found by maximizing the power output, $P_{\max }=I_{\mathrm{opt}} \times U_{\mathrm{opt}}$. This is obtained by constructing the I-V characteristics, using eq. (1), by varying the relative positions of the electrochemical potentials. 

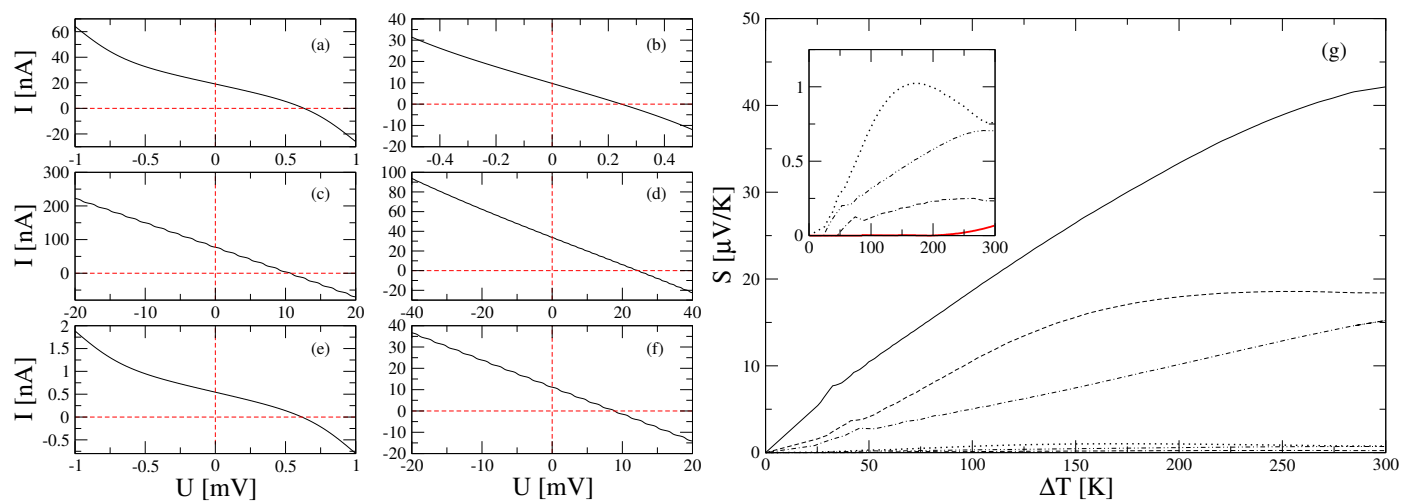

Figure 3. I-V characteristics of the hybrid nanoribbon systems (a-f), for the structures indicated in Fig. 1, at $T=300 \mathrm{~K}$. Thermopower (absolute value) vs. $\Delta T$, at the optimum working point, for each structure: (a) dotted, (b) dash-dash-dotted, (c) dashed, (d) solid, (e) dot-dot-dashed, (f) dot-dashed. The inset contains a detailed view over the samples with lower thermopower $(\mathrm{a}, \mathrm{b}, \mathrm{e})$ and the data corresponding to the pristine graphene (thick solid line) is represented.

\section{Results and discussion}

The spin dependent transmission functions are presented in Fig. 2 for each considered structure. Figure 2(a) shows also the case of a pristine graphene nanoribbon, where the step-wise transmission function presents pronounced maxima, with up to four modes for each spin channel [26]. Around the Fermi energy the transmission function is constant, with only one conducting mode per spin channel. Pristine zig-zag graphene nanoribbons have intrinsic anti-ferromagnetic (AFM) coupling between the edges in the ground state. However, the spin state of the edge atoms can be tuned in a ferromagnetic (FM) configuration by applying a magnetic field [25]. In the FM configuration the zig-zag nanoribbons are metallic even in the case of very narrow widths, a high conductance being important for achieving a high figure of merit.

A transmission function which presents little variation around the chemical potential is not desired for thermoelectric applications. Therefore using pristine zig-zag nanoribbons the obtained thermopower is rather low. One option to improve the thermoelectric properties is by introducing substitutionally boron and nitrogen atoms, forming atomic sized $\mathrm{hBN}$ domains. The $\mathrm{B}-\mathrm{N}$ sequences introduce barriers in the otherwise conductive graphene nanoribbon, which bring variations in $\mathcal{T}(E)$. As test cases, nanoribbon structures with lateral barriers, thin tunneling double barriers and graphene dot systems are constructed.

The spin dependent transmission functions become more and more asymmetric by increasing the number of B-N substitutions. The two spin channels are scattered differently, the up-spin component, parallel with spin polarized edges, being transmitted by a larger amount compared to the down-spin component. Furthermore, as expected the presence of larger BN domains reduces overall the total transmission function. These two factors, namely the increase of asymmetry in $\mathcal{T}(E)$ with respect to the chemical potential and a possible decrease in electrical conduction, should be taken into account in designing performant thermoelectric devices.

Next, the I-V characteristics are presented in Figure 3(a-f). It was found that the double barrier systems (c,d) have the largest $U_{\mathrm{oc}}$ and $I_{\mathrm{sc}}$ and, given the similar linear shape, the maximum power output. The thermopower at the optimum working point is also the largest for these two systems, $42 \mu \mathrm{V} / \mathrm{K}$ 
and $18 \mu \mathrm{V} / \mathrm{K}$, respectively, as shown in Fig. 3(g), followed by one of the graphene-dot systems, labeled as system (f), with $15 \mu \mathrm{V} / \mathrm{K}$. The values were determined by setting $T_{\mathrm{L}}=10 \mathrm{~K}$ and varying $\Delta T$. By comparison, the pristine zig-zag graphene exhibits a very poor thermoelectric effect, visible in the inset of Fig. 3(g), with a maximum value of only $0.05 \mu \mathrm{V} / \mathrm{K}$.

\section{Conclusions}

The thermoelectric effects of several hybrid graphene - hBN nanoribbon structures were investigated using DFT calculations. The pristine zigzag graphene nanoribbon presents a quite small thermopower, as the transmission function is flat around the chemical potential. By introducing boron and nitrogen substitutions, the transmission functions become more asymmetric resulting in an enhanced Seebeck coefficient. Although there are many options for introducing these types of asymmetries, e.g. by functionalizing the graphene nanoribbons, hBN has the advantage of being well integrated in the graphene structure. Amongst the structures investigated, the double barrier systems are most promising for thermoelectric applications. However, the optimal configuration of the hBN domains within graphene nanostructures remains to be determined.

\section{Acknowledgements}

This work was supported by the National Ministry of Education (MEN) and the Romanian Executive Unit for Financing Higher Education, Research and Innovation (UEFISCDI) under projects PN-II-ID-PCE-2011-3-0960 and PN09370104/2015 and from the Hulubei-Meshcheryakov Programme, JINR Order 34/23.01.2015, p.97.

\section{References}

[1] Alexandra Witze, Nature 515, 484 (2014)

[2] P. Dollfus, Viet Hung Nguyen and J. Saint-Martin, J. Phys.: Condens. Matter 27, 133204 (2015)

[3] A. A. Balandin, S. Ghosh, W. Bao, I. Calizo, D. Teweldebrhan, F. Miao and C. N. Lau, Nano Lett. 8, 902 (2008)

[4] H. Sevincli, C. Sevik, T. Cagin and G. Cuniberti, Sci. Rep. 3, 1228 (2013)

[5] B. K. Nikolic, K. K. Saha, T. Markussen and K. S. Thygesen, J. Comput. Electron. 11, 78 (2012)

[6] W. Oswald and Z. Wu, Phys. Rev. B 85, 115431 (2012)

[7] X.-M. Wang, D.-C. Mo and S.-S. Lu, J. Chem. Phys. 138, 204704 (2013)

[8] T. Ouyang, Y. Chen, L.-M. Liu, Y. Xie, X. Wei and J. Zhong, Phys. Rev. B 85, 235436 (2012)

[9] G. A. Nemnes and Camelia Visan, Comput. Mat. Sci. 109, 14 (2015)

[10] Li Song, Lijie Ci, Hao Lu et al., Nanolett. 10, 3209 (2010)

[11] Yongji Gong, Gang Shi, Zhuhua Zhang et al., Nature Commun. 5, 3193 (2014)

[12] Z. Liu et al, Nanotechnol. 8, 119 (2013)

[13] L. Britnell, R. V. Gorbachev, A. K. Geim, L. A. Ponomarenko, A. Mishchenko, M. T. Greenaway, T. M. Fromhold, K. S. Novoselov, L. Eaves, Nature Commun. 4, 1794 (2013)

[14] V. H. Nguyen, F. Mazzamuto, A. Bournel and P. Dollfus, J. Phys. Appl. Phys. 45, 325104 (2012)

[15] G. Fiori, A. Betti, S. Bruzzone and G. Iannaccone, ACS Nano 6, 2642 (2012)

[16] G. A. Nemnes, J. Nanomater. 2012, 748639 (2012)

[17] G. A. Nemnes and S. Antohe, Mater. Sci. Eng. B 178, 1347 (2013) 
[18] Camelia Visan, Rom. Rep. Phys. 66, 983 (2014)

[19] A. A. Nila, G. A. Nemnes, A. Manolescu, Rom. J. Phys. 60, 696 (2015)

[20] K. Yang, Y. Chen, R. D’Agosta, Y. Xie, J. Zhong, and A. Rubio, Phys. Rev. B 86, 045425 (2012)

[21] Camelia Visan, J. Electron. Mater. 43, 3470 (2014)

[22] Y. S. Liu, W. Q. Zhou, J. F. Feng, X. F. Wang, Chem. Phys. Lett. 625, 14 (2015)

[23] J. M. Soler, E. Artacho, J. D. Gale, A. Garca, J. Junquera, P. Ordejon, and D. Sanchez-Portal, J. Phys. Cond. Mater. 14, 2745 (2002)

[24] M. Brandbyge, J.-L. Mozos, P. Ordejon, J. Taylor, and K. Stokbro, Phys. Rev. B 65, 165401 (2002)

[25] X.F. Yang, H.L. Wang, X.K. Hong, Y.S. Liu, J.F. Feng, X.F. Wang, C.W. Zhang, F. Chi, M.S. Si, Organic Electronics 24, 80 (2015)

[26] An Lipin and Liu Nianhua, J. Semicon. 32, 052001 (2011) 